3 Benjamin G. Farrar ${ }^{1,5^{*}}$, Drew M. Altschul ${ }^{2}$, Julia Fischer ${ }^{3}$, Jolene van der Mescht ${ }^{2}$, Sarah Placì ${ }^{3}$,

\section{cognition research.}

\title{
Claims and statistical inference in animal physical
} Camille A. Troisi ${ }^{4}$, Alizée Vernouillet ${ }^{1}$, Nicola S. Clayton ${ }^{1}$, and Ljerka Ostojić ${ }^{5}$

5

6

${ }^{1}$ Department of Psychology, University of Cambridge, Cambridge, UK

7

${ }^{2}$ The University of Edinburgh, Edinburgh, UK

8

${ }^{3}$ Cognitive Ethology Laboratory, German Primate Center, Kellnerweg 4, 37077, Göttingen, Germany

9

${ }^{4}$ School of Biological, Earth and Environmental Sciences, University College Cork, Cork, Ireland

10

${ }^{5}$ Institute for Globally Distributed Open Research and Education (IGDORE)

11

12

*Corresponding author (Email: bgf22@cam.ac.uk)

13

14 
15 Abstract - Twenty years after Povinelli's "Folk Physics for Apes", this paper assesses how researchers have made claims about animal physical cognition, and the statistical inferences that have been used to support them. These data are relevant in light of the current replicability issues facing science. We surveyed 116 published experiments from 63 papers on physical cognition, which included data from 43 different species of animals. Across these experiments most sample sizes were small, with often fewer than 10 animals being tested. However, in contrast to related psychological disciplines, we found that only $62 \%$ of our sample of physical cognition research made positive claims. This suggests that animal physical cognition does not have a strong publication bias towards positive results. Furthermore, we found evidence that researchers are making many true statistical inferences at the individual level, i.e. whether individual animals pass certain tests of physical cognition or not. In contrast, the strength of evidence of statistical effects at the group level was weaker and consistent with many effect sizes being overestimated. Overall, our analysis provides a cautiously optimistic analysis of reliability and bias in animal physical cognition research, however it is nevertheless likely that a non-negligible proportion of results will be difficult to replicate.

KEYWORDS: Physical cognition, folk physics, evidence, statistical inference, publication bias 
Povinelli's (2000) "Folk Physics for Apes" has had a lasting impact on the study of

what animals understand about the physical world, their "folk physics". However, in "Folk Physics for Apes", Povinelli also highlighted many of the issues that have been foregrounded in science's current replication crisis (see Flis, 2019), such as replication (Open Science Collaboration, 2015; Povinelli, 2003, p. 16; Zwaan, Etz, et al., 2018) falsification (LeBel et al., 2017), strong inference (Chamberlin, 1965; Open Science Collaboration, 2015; Platt, 1964; Stevens, 2017) and being willing and able to interpret negative results (Fanelli, 2012; Daniël Lakens, 2017; Povinelli, 2003, p. 50; Rosenthal, 1979). In the current paper, we use data from published experiments in animal physical cognition in order to explore how the field has used statistical inferences to make claims, in light of the current replicability issues science faces.

Since many scientific results cannot be replicated consistently (Baker, 2016; Begley \& Ellis, 2012; Camerer et al., 2018) there is a growing body of meta-research attempting to analyse what features might distinguish between reliable and unreliable literatures (Ioannidis, 2018). Publication biases, a lack of replication studies and "low power" or many just significant p-values may be indicators of less reliable research (Benjamin et al., 2018; Button et al., 2013; Makel et al., 2012; Nissen et al., 2016; Rosenthal, 1979; Sena et al., 2010; Simonsohn et al., 2014; Zwaan, Etz, et al., 2018), whereas routinely publishing negative results, replicating results and providing strong statistical evidence of effects are indicators of more reliable research (Smith \& Little, 2018; Zwaan, Pecher, et al., 2018). However, to date, most of these studies have focused on human psychology, and relatively little research has been conducted on animal cognition. This situation exists despite some areas of animal cognition research sharing their research methods with disciplines that are struggling to replicate results (Farrar et al., 2020; Maes et al., 2016; Poulin-Dubois et al., 2018). The current study therefore attempts to provide data on several features of reliability from across the animal physical cognition literature. First, we document the prevalence of positive claims in the physical cognition 
literature, which we define as claims of either the presence of a more exceptional ability in the animal or species, a novel effect, or the animal(s) "passing" a test. This proportion of positive claims can provide an indirect measure of publication and theoretical biases in animal physical cognition research, based on the assumption that literatures that contain near exclusively positive claims likely have a complement of unpublished negative reports and/or preferentially interpret the results that are published in a positive direction.

Second, we attempt to explore how researchers make the statistical inferences that support their claims, and the strength of evidence that these statistical inferences provide. Specifically, we explore the distribution of significant $p$-values that support researchers' claims from across our sample of physical cognition studies. By comparing the shape of a research body's $p$-value distribution to the shape of $p$-value distributions expected under different conditions, some information can be extracted regarding the strength of evidence provided against null hypotheses across this research (Lakens, 2017; Simonsohn et al., 2014). For example, if an observed $p$-value distribution is more similar to a $p$-value distribution consistent with studying only false effects (H0 always true) than a $p$-value distribution expected if researchers were studying exclusively true effects with $80 \%$ power, then this could indicate that the group of research as a whole might be unreliable.

When investigating the $p$-value distributions from the physical cognition literature, we distinguish between two types of statistical inference that are routinely used in animal cognition; group-level inferences and individual-level inferences. Group-level inferences attempt to characterise the population of interest, for example, "do dogs look longer at physically impossible events than physically possible events?" or "do chimpanzees pass the trap tube task?". Their results are used to generalise to the population from which the sample of dogs was drawn. In contrast, individual-level inferences estimate the parameters for individual animals only, for example, “does Rex the dog look longer at physically impossible 
events than physically possible events?", or "does Megan the chimpanzee pass the trap tube task?". While both population-level and individual-level statistical inferences are routinely made in animal physical cognition studies, they differ in what their results tell us. Specifically, group-level inferences generalise from the sample of individuals to the population they were drawn from, whereas individual-level inferences generalise from the sample of trials to the population of trials within that individual. Hence, individual-level inferences may be more suitable to support claims of the form: "in principle, species X can perform behaviour Y", whereas group-level inferences may be more suitable to support claims of the form: "species X performs behaviour Y'. However, the prevalence of each of these types of claim across the animal physical cognition literature is unknown, and so this paper attempts to document just how frequently both inferences are made and to what extent they produce different $p$-value distributions. These questions are interesting as often only a minority of animals pass a given test of physical cognition, yet they appear to do so convincingly. For example, when learning the trap tube task in Povinelli's original work, Megan was correct on 80/100 trials, ( $p=.000000000135)$ yet she was the only chimpanzee to do so. Hence, it is possible that researchers can generate strong evidence of effects at the individual-level, without there being clear group-level effects. To probe this further, we also coded the number of animals reported to have "passed" each test of physical cognition. In summary, this paper provides data on the types of claims and statistical inferences used across the animal physical cognition literature. These features are listed in Table 1. 
Table 1: The data we coded as part of this study, relating to claims and statistical inferences in the animal physical cognition literature.

\begin{tabular}{|c|c|}
\hline Data & Description \\
\hline $\begin{array}{l}\text { Main claim and whether it } \\
\text { was supported }\end{array}$ & $\begin{array}{l}\text { What the main claim of the paper was, and whether it was "positive", } \\
\text { i.e., claiming either the presence of a more exceptional ability in the } \\
\text { animal(s), a novel effect, or the anima(s) "passing" a test; "negative", } \\
\text { i.e., claiming either the absence of a more exceptional ability in the } \\
\text { animal(s), a novel effect, or the animal(s) "failing" a test; or } \\
\text { "inconclusive", i.e., claiming neither the presence or absence of a } \\
\text { more exceptional ability in the animal(s), a novel effect or the } \\
\text { animal(s) passing or failing a test. }\end{array}$ \\
\hline Hypothesis level & $\begin{array}{l}\text { Whether the authors' main claims are based on individual-level or } \\
\text { group-level inferences, or both. }\end{array}$ \\
\hline Statistical result and p-value & $\begin{array}{l}\text { The reported } p \text {-value for the critical statistical test (i.e., the statistical } \\
\text { test that supported the main claim of the paper). If the } p \text {-value was } \\
\text { reported as an inequality, e.g. } p<0.05 \text {, we calculated the exact } p \text { - } \\
\text { value from the information in the text, if sufficient information was } \\
\text { available. }\end{array}$ \\
\hline $\begin{array}{l}\text { Sample Size and N passing } \\
\text { each test }\end{array}$ & $\begin{array}{l}\text { For exploratory purposes, we coded the number of animals tested in } \\
\text { the experiment, and, if individual-level analyses were used, the } \\
\text { number of animals "passing" a test. }\end{array}$ \\
\hline
\end{tabular}


110

\section{Methods}

The working introduction and methods for this paper were deposited before data collection at osf.io/a6cu7/registrations.

\section{Paper Inclusion}

This project attempted to code information from 200 published experiments in animal physical cognition studies. Papers were found by a keyword search in Scopus. Papers with titles, abstracts and/or keywords containing the following keywords: "folk physics" OR "physical cognition" were searched for, which returned 167 results on 26th November 2019. An error in an earlier search meant we had expected over 600 results from this search, and so after realising the search returned only 167 results, we performed a further search for "traptube" OR "trap tube" OR "trap table" OR "trap-table", which returned a further 58 results. Papers were listed by 'relevance', and the titles and abstracts of each paper were then screened for whether they fit our inclusion criteria: being a study of physical cognition in captive animals, including animals transiently kept in captivity for testing. For multi-experiment papers and papers with many different conditions, two experiments or conditions were randomly selected and coded. This was decided in order to give greater representation to common designs used in animal cognition studies, while minimizing how affected our analysis would be by nonindependence in the data points.

Of the 167 papers from the first search, 60 were coded as fitting the inclusion criteria, i.e. as experiments on physical cognition in captive animals. An extra 12 non-duplicate experiments from the trap-tube/trap-table search fitted the inclusion criteria and were added to the sample. A second screen of the full papers then led to 9 more studies being excluded: 7 for not containing experiments on physical cognition or being developmental or individual differences studies, 1 for us being unable to access the paper, and 1 for the paper not being in 
English. Of the remaining 63 papers, 53 had multiple physical cognition experiments, from which we randomly selected 2 to be coded. This produced a total sample of 116 experiments from 63 papers.

\section{Coding Protocol}

The coding protocol detailed exactly what was coded from the literature in order to measure the features listed in Table 1. The exact protocol can be found at https://osf.io/wkpeq/. To refine the protocol and assess the feasibility of the study, an early version of the protocol was piloted on the first experiments of Povinelli's "Folk Physics for Apes", and a second protocol piloted on four extra studies. The pilot studies revealed that our original coding plan was too complicated, and we therefore limited the scope of the coding to include only the six measures reported in the paper.

For each paper, two coders coded the following features:

1. The main claim of the paper, coded from the abstract of each paper

2. Whether this claim was "positive", "negative" or "inconclusive"

3. The text of the primary group-level statistical inference made supporting the main claim, if applicable

4. The text of the primary individual-level statistical inference made supporting the main claim, if applicable. If multiple individual-level inferences were made supporting the main claim, we coded the first one presented.

5. The sample size

6. The number of animals "passing" a test, if applicable

BGF then coded the exact $p$-values from the texts of the group- and individual-level statistical inferences that were coded. For 44 experiments where non-exact values were reported, e.g. $p<.05$, BGF then calculated them from the reported test statistics, if sufficient 
information was available. We additionally planned to assess how researchers interpreted nonsignificant $p$-values, however we decided that the number of non-significant $p$-values that we coded (16) was insufficient for any representative analysis.

\section{Coding and Reliability}

Coders were trained on four pilot studies, and any disagreement in this coding phase was used to refine the protocol. All 116 experiments of our final sample were double coded: BGF coded all of the experiments, and CAT, DA, JF and JvdM acted as second coders. Where there was disagreement between the double-coded items, this was resolved by a third coder by reference to the coding protocol (AV, LO and SP). For all disagreements, the third coder's decision was used in the final dataset, and in the 3 cases where the third coder's decision disagreed with both the first and second coder, the third coder's choice was retained. For the three variables that were coded by copying and pasting text, these were coded as agreeing if there was substantial overlap between the content of both coders, decided by BGF. This changed from the criteria we archived in our working methods, which was that the coders agreed if $50 \%$ of the text overlapped. We changed this criterion as the coders often varied in how much text they included, despite focusing on the same claim or inference. All of the data, including reliability decisions, and code, are available at https://osf.io/wkpeq/.

Analyses

We first present descriptive data and visualisations of the following information: the distribution of species and groups from our sample, the sample sizes across the experiments, the frequency of positive and negative claims, the frequency of group-level and individuallevel inferences, and $p$-value distributions for both group-level and individual-level inferences. We then used these data to explore the types of claims and strength of statistical inferences present in the animal physical cognition literature. We do the latter by qualitatively comparing the $p$-value distributions of the group-level and individual-level data with simulated 
distributions from researchers studying only true statistical effects with $80 \%$ power, or studying zero true effects, i.e. $5 \%$ power (for simulation details, see Appendix). We do not make strong or quantitative conclusions from these data due to their non-independence and uncertainty about the theoretical $p$-value distributions under the different hypothetical scenarios $(80 \%$ power, $20 \%$ power and $5 \%$ power). We explain this uncertainty in the Discussion, as seeing the data first can help understand the limitations of the analysis.

\section{Results}

\section{Coding reliability}

The first two coders agreed on 56 out of 63 claims from each paper's abstract, Cohen's $K=0.89$, and agreed on 38 of 63 of their levels, i.e. whether the claims were positive, negative or inconclusive. This agreement rate was slightly lower than anticipated, however all but two of the disagreements occurred when one of the coders labelled a claim "inconclusive" and the other labelled it as either "positive" or "negative". Accounting for the ordinal structure of the data, in which a disagreement between "positive" and "negative" is more severe than a disagreement between "positive" or "negative" and "inconclusive", Cohen's $K_{\text {weighted }}=0.47$. Coders agreed on 45 of 75 group-level inferences, Cohen's $K=0.60$, and 63 of 93 individuallevel inferences, Cohen's $K=0.68$. Much of the disagreement here came from a conflicting interpretation of what to code as a group-level vs individual-level inference between two of the coders. Excluding the data from these two coders, which was rectified during coding by a third coder, agreement rose to Cohen's $K_{\text {group }}=0.66$ (32 of 48), Cohen's $K_{\text {individual }}=0.82$ (56 of 68). Sample sizes were coded equally in 103 of 116 experiments, Cohen's $K=0.89$, and the number of animals passing each test was agreed in 56 of 94 , Cohen's $K=0.59$. All disagreements were then resolved by a third coder to produce the dataset we used in the analysis. 
While coders agreed on most measures most of the time, there was still substantial

207

208

209

210

211

212

213

214

215

216

217

218

219

220

221

222

223

224

225

226 variation in how they coded the claim levels (positive, negative or inconclusive), as well as the group-level and individual-level inferences. This disagreement likely stems from three sources. First, and particularly relevant for the claim levels, is that the coding is inherently subjective and as a consequence there are no verifiably correct or incorrect answers. Nearly all disagreements came from one coder suggesting the claim was "inconclusive" whereas the other suggested it was either "positive" or "negative". These disagreements may have occurred due to a genuine ambiguity in the wording of the claims, or alternatively, when different coders had different interpretations of what constituted a positive or negative claim from our coding manual. Indeed, many disagreements in the group- and individual-level inference texts appear to be due to coders disagreeing on the definition of each type of inference, which was rectified through coding by a third coder. Second, we used a large total number of coders (8), and this heterogeneity should have protected against unintentional individual researcher bias, although BGF was a coder on all papers. Finally, it is likely that a small number of transcription errors also have contributed to disagreements between coders.

\section{Species and Sample Sizes}

There were 45 different species represented across the 63 physical cognition papers that we coded (Figure 1), with chimpanzees (8), New Caledonian crows (8), dogs (7) and keas (6) being the most common. Notably, our search protocol of "folk physics" OR "physical cognition", and a variety of trap-tube and trap-table searches, returned no species outside of mammals and birds. 


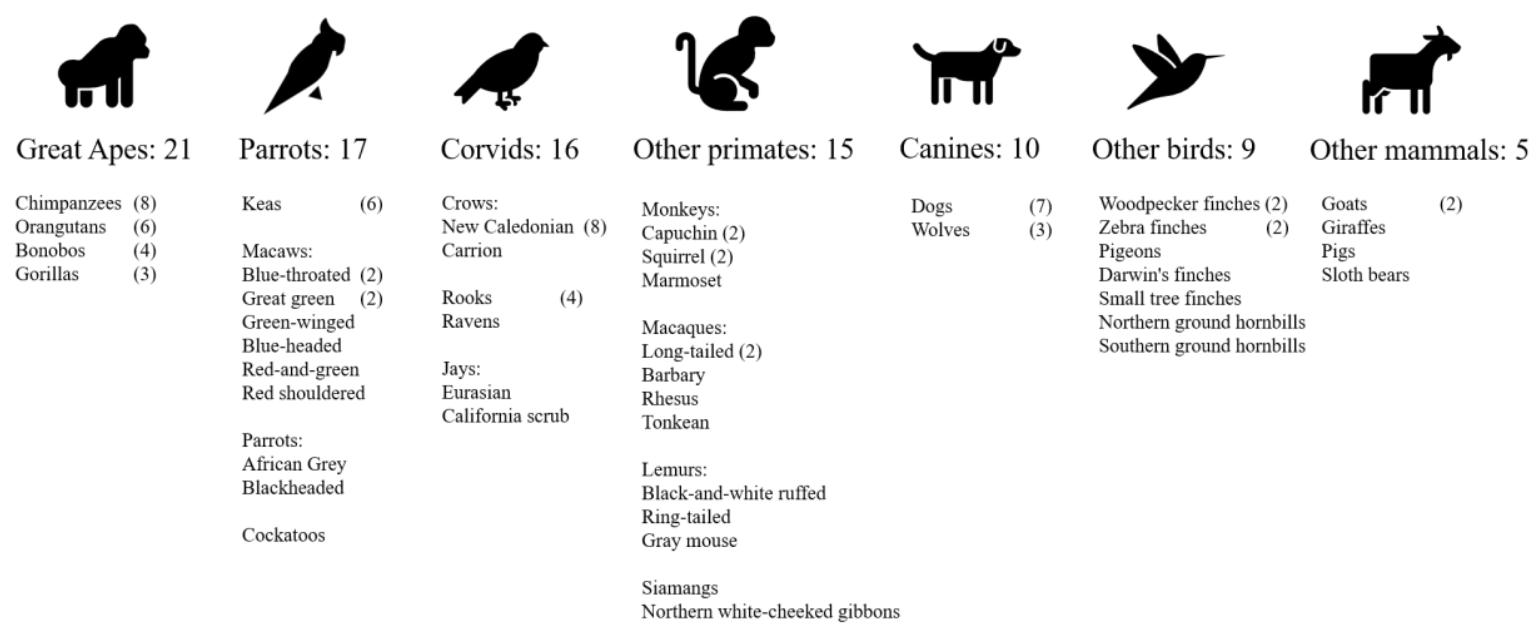

Figure 1: The number of papers investigating each species in our sample of physical cognition papers, e.g. there were 8 papers that included chimpanzees in the sample, and two papers including goats. The sample consisted of behavioural experiments on captive animal physical cognition, and was sampled using a Scopus search for "folk physics" OR "physical cognition", and a variety of trap-tube and traptable combinations.

Sample sizes ranged from 1 to 56, with a median of 7 (Figure 2). The relatively large number of experiments with $\mathrm{N}<5$ is partly due to us including 8 transfer tasks in our sample, in which only a subset of the original sample was tested. There was some evidence of speciesspecific differences in sample sizes, for example all sampled corvid studies had a sample size of fewer than 10 individuals, whereas studies with canines and non-great ape primates often had larger samples. 


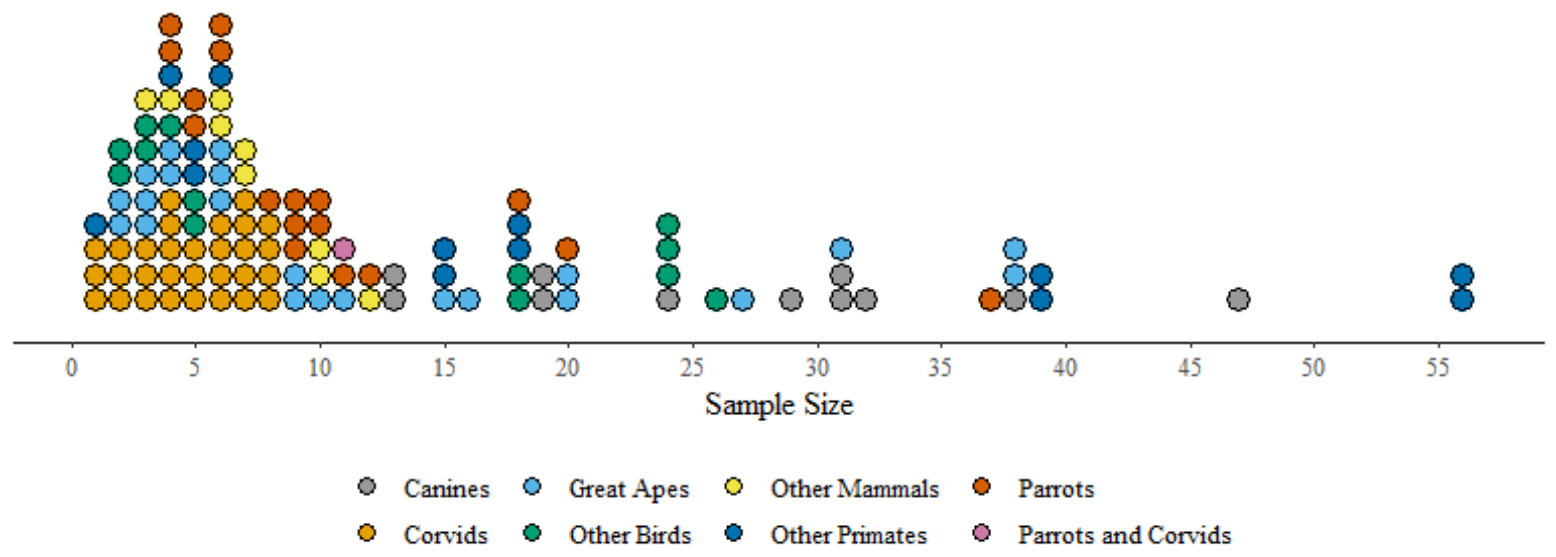

Corvids Other Birds Other Primates 0 Parrots and Corvids

236 Figure 2: The distribution of the total sample sizes from each experiment of our sample of physical

237 cognition experiments. NB: data points are not independent as some of the sample sizes are from

238 experiments from the same papers, and some of the papers are from the same laboratories that tested

239 the same sets of captive animals.

240 Claims and inferences

241 Of the 63 papers, $39(62 \%)$ made positive claims, i.e. claimed either the presence of a 242 more exceptional ability in the animal, a novel effect, or the animal(s) "passing" a test. Fourteen $243(22 \%)$ papers made negative claims, i.e. claimed either the absence of a more exceptional 244 ability in the animal, a novel effect, or the animal(s) "failing" a test. Ten (16\%) papers made 245 inconclusive claims, i.e. claims that asserted neither the presence or absence of a more exceptional ability in the animal(s). While our sample size was small for each group, the number of positive, negative and inconclusive results did not seem to differ largely across groups, with the possible exception of corvids for which there were 13 positive, 2 inconclusive and 0 negative claims (Table 2). 
Table 2: The number of each type of claim (positive, negative or inconclusive) made in the sample of physical cognition papers by study group. The group "Parrots and Corvids" comes from a single paper that studied both groups.

\begin{tabular}{|c|c|c|c|}
\hline Group & Positive & Negative & Inconclusive \\
\hline Canines & 3 & 3 & 1 \\
\hline Corvids & 13 & 0 & 2 \\
\hline Great Apes & 9 & 2 & 1 \\
\hline Other Birds & 6 & 3 & 1 \\
\hline Other Mammals & 4 & 1 & 1 \\
\hline Other Primates & 6 & 2 & 1 \\
\hline Parrots & 8 & 3 & 2 \\
\hline Parrots and corvids & 0 & 0 & 1 \\
\hline
\end{tabular}

From the 63 papers, the main claims were supported by 68 group-level and 88 individual-level inferences, with 24 papers including both group-level and individual level statistical inferences. Across the 116 experiments sampled from the 63 papers, 40 experiments included both group- and individual-level inferences in support of their claims, 28 used only group-level inferences and 48 only individual-level inferences. $p$-values

From the 68 group-level inferences, we had sufficient information to extract 58 exact $p$-values, of which 46 were $<.05$. From the 88 individual-level inferences, we had sufficient information to extract 49 exact $p$-values, of which 41 were $<.05$. The density distributions of these significant $p$-values are plotted in Figure 3, alongside simulated distributions from research with $80 \%$ power to detect a true effect (" $80 \%$ power simulation", uppermost plot), 
research with $20 \%$ power to detect a true effect (“20\% power simulation", second from top), research where all null hypotheses were true ("False positive simulation", third from top).

$80 \%$ power simulation

$20 \%$ power simulation
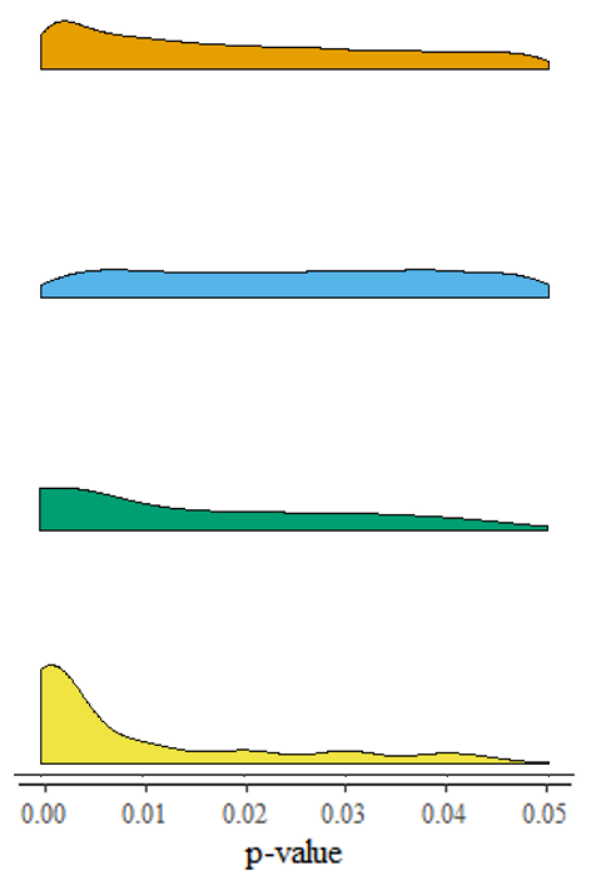

False positive simulation

Physical cognition sample: Group

Physical cognition sample: Individual

Figure 3: Density distributions of significant $p$-values resulting from simulated research with H1 always correct and research performed with $80 \%$ power (uppermost plot), $20 \%$ power (second panel from top), and $\mathrm{H} 0$ always correct (third from top), the group-level inferences from our sample of physical cognition research ( $\mathrm{N}=46$, fourth panel from top) and the individual-level inferences from our sample of physical cognition research $(\mathrm{N}=41$, bottom panel).

Both the group- and individual-level statistical inferences have the largest density of $p$ values between 0 and 0.01 , providing evidence of correct rejections of $\mathrm{H} 0$. This pattern is very apparent for the individual-level inferences, which is consistent with research performed at 
relatively high power. In addition, the group-level distribution looks similar to the $20 \%$ power distribution and could reasonably be modelled as a mixture of low power research and some higher power and false positive research.

\section{Animals passing each test}

Of the 88 experiments making individual-level inferences, we were able to code the number of individuals who "passed" the experimental test from 87 . Zero individuals passed the test in 15 experiments, the maximum number of individuals passing a test was 17 and the median was 3 . The number of individuals passing each test, and the corresponding original sample size are plotted in Figure 4 (left panel). Further, the proportion of animals passing the experimental tests varied substantially between experiments, from 0 ( 15 experiments) to all (25 experiments). The median proportion of animals passing each experimental test was 0.6 (Figure 4 , right panel).
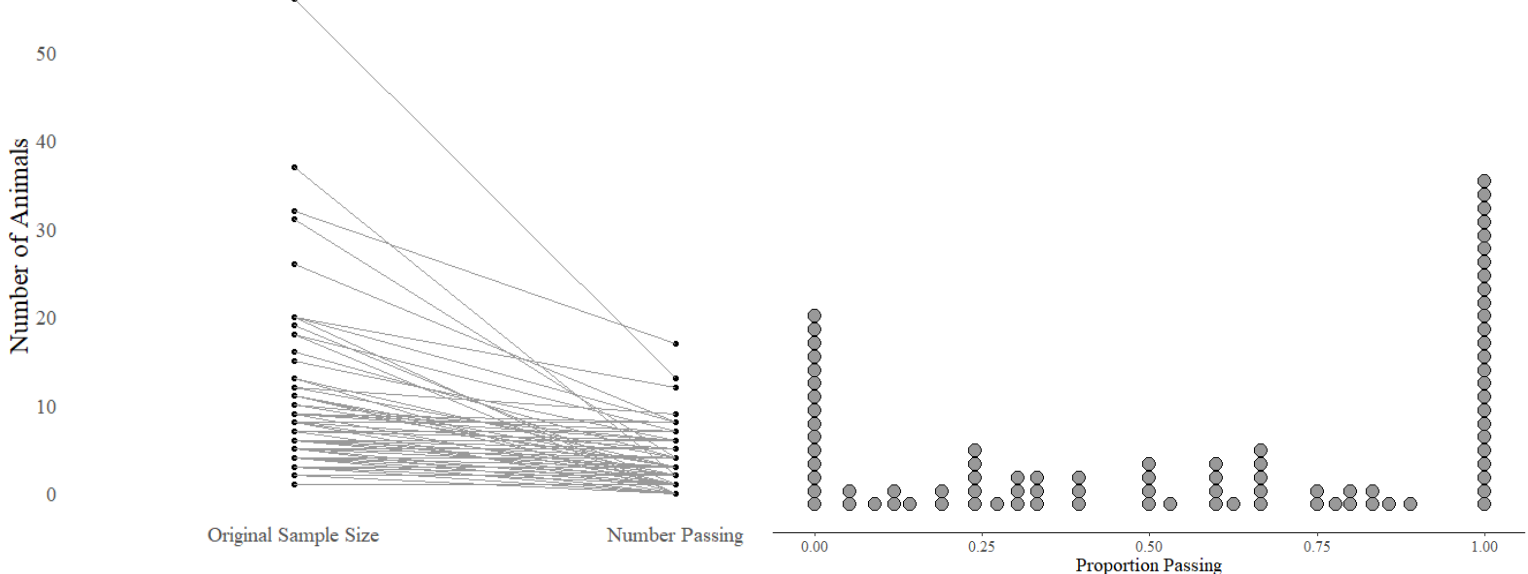

Figure 4: The number of animals passing each experimental test, and the corresponding original sample size from our sample of physical cognition research (left panel). The proportion of animals passing the test in each experiment from our sample of physical cognition research (right panel). 
293

294

295

296

297

298

299

300

301

302

303

304

305

306

307

308

309

310

311

312

313

314

315

316

\section{Discussion}

\section{Claims, Sample Sizes and "Passing A Test"}

We found that $62 \%$ of claims made in our sample of animal physical cognition papers were positive, $22 \%$ were negative and $16 \%$ were inconclusive. This figure of $62 \%$ positive claims is lower than previous reports of $\sim 90 \%$ positive claims in psychology in general (Fanelli, 2010), and closer to the $\sim 50 \%$ of positive findings from novel Registered Report studies in psychology (Scheel et al., 2020). This suggests that animal physical cognition research in general may not have an intense bias towards positive claims and positive results, and that animal physical cognition research is receptive to negative reports. Furthermore, papers often reported the results of non-significant statistical tests at both the group and individual level. These findings are encouraging, however they may be a consequence of a separate issue in animal physical cognition research: having "benchmark" tasks for certain abilities (see Eaton et al., 2018). When a task reaches 'benchmark' status for a cognitive ability, for example the trap-tube task or mirror-mark task, positive performance on the task can be conflated with the presence of the cognitive ability and negative performance on the task can be conflated with the absence of the cognitive ability. And in contrast to novel tasks, researchers, reviewers and editors may be less likely to critically evaluate to what extent the benchmark test assesses the ability of interest, i.e., its construct validity. As such, once a task becomes established, it can become an easy target to generate publications, independent of the informativeness of the data it can produce.

\section{Strength of evidence}

Finally, our $p$-value distribution analysis provides support for the hypothesis that researchers are generating stronger evidence against null hypotheses for individual- rather than group-level statistical effects (Figure 3). While the $p$-value distribution for individual-level 
inferences seems largely consistent with many relatively high-powered tests at the individual level, this was less of the case for the group-level inferences. While there were still more low $(<0.01)$ than high $(0.04-0.05) p$-values in the group data, this distribution could be modelled as a combination of a set of low powered studies with some false positives and some highpower studies included (Figure 5). This distribution does not seem consistent with a system of high-powered research. As a consequence, we should expect that many effect sizes from positive group-level inferences are overestimated, sometimes considerably (Farrar et al., 2020; Hedges, 1984).

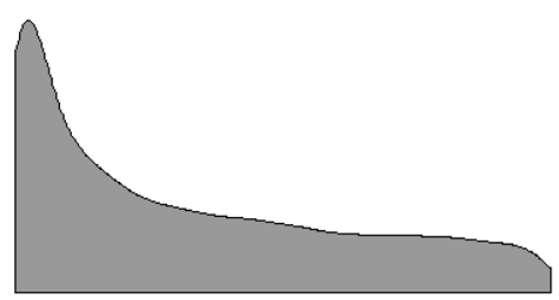

$1 / 4$ false positive, $2 / 320 \%$ power, and $1 / 1280 \%$ power simulation
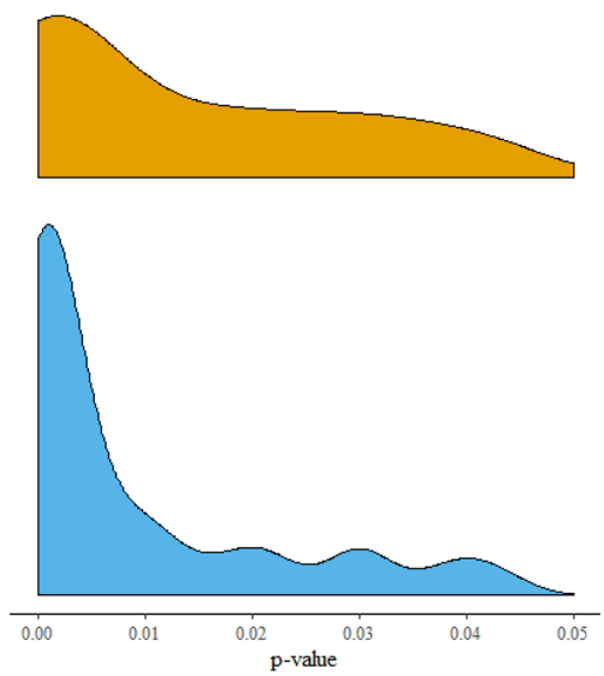

Physical cognition sample: Group

Physical cognition sample: Individual

Figure 5: Density distributions of significant $p$-values resulting from simulated research with a combination of $1 / 1280 \%$ power $2 / 320 \%$ power and $1 / 45 \%$ power (uppermost panel), the group-level inferences from our sample of physical cognition research $(\mathrm{N}=46$, middle panel) and the individuallevel inferences from our sample of physical cognition research $(\mathrm{N}=41$, bottom panel). 
However, there are several caveats to this $p$-value distribution analysis. First, the sample sizes are not large, with $\mathrm{N}=46$ and $\mathrm{N}=41 p$-values for the group and individual-level statistical inferences respectively. Hence, the distributions will easily be influenced by including or excluding extra $p$-values. Second, the simulated distributions come from $t$-tests on normally distributed data, which produce uniform distributions when the null hypothesis is true, and exponential curves when $\mathrm{H} 0$ is false, like the one shown in Figure 3 under $80 \%$ power. However, in animal cognition research there are often many tests performed with small sample non-parametric or binomial tests, which result in non-normal $p$-value distributions under the null. Nevertheless, aside from quite rare occurrences (e.g. binomial tests with $\mathrm{N}=6$ and $\mathrm{p}=0.5$ or Wilcoxon-signed rank tests with $\mathrm{N}=6$ ), the $p$-value distributions under $\mathrm{H} 0$ soon approximate a uniform distribution, and an exponential distribution under H1. Our observed distribution for the individual level inferences, in which there were many $p$-values following significant binomial tests from 10 trials demonstrates this. Hence, while the simulated distributions assume that there are no small-sample non-parametric or binomial tests, that this assumption is false does not invalidate $p$-value distributions as a useful method to investigate how frequently a research area on average provides strong evidence against null hypotheses. Furthermore, our $p$-value distribution analysis provides very little information about individual studies themselves. This is because the studies do not share the same reference class, i.e. they are not randomly sampled from the same underlying distribution. Some effects are very likely to be true positives, and some very likely to be false positives, and $p$-value distributions do not account for this. Hence, when evaluating individual studies, their own features such as statistics, test design and plausibility of hypothesis, provide the key information on whether a given effect is estimated accurately, rather than a $p$-value distribution in which the majority of $p$-values will be irrelevant to the claim at hand. 
354

\section{Limitations}

There are two main limitations of our sampling and analyses to consider. First, our sampling strategy did not produce a random sample of animal physical cognition experiments, instead the sample was drawn from Scopus key word search for "folk physics" OR "physical cognition" and "trap-tube" OR "trap tube" OR "trap table" OR "trap-table" in November 2019. As such, our sample may not be fully representative sample of animal physical cognition research in general. Perhaps, for example, studies mentioning "physical cognition" tend to make more positive claims than those that do not, or individual researchers might be more or less likely to use the phrase "folk physics", causing them to be over or under-represented in our sample, respectively. Second, the data points in our analysis come from non-independent experiments, i.e. experiments from the same paper, laboratory or on the same animals, and this may lead to the slight over-representation of some designs in our analysis.

\section{Conclusions}

The present study analyzed 116 animal physical cognition experiments from 63 papers.

Our sample contained $62 \%$ positive claims, which suggests that animal physical cognition research does not have strong bias towards positive claims, unlike many other fields in science. These findings are encouraging, however they may be a product of animal physical cognition having many "established" tasks. Such tasks, like the trap-tube task, can become easy targets to generate publications with either positive or negative results, independent of the informativeness of the data it can produce. Finally, our $p$-value analysis suggests that researchers are finding many true statistical effects at the individual level, however many published group-level effects in animal physical cognition research may be overestimated; and this result may generalise to physical cognition and animal cognition research more generally. 

physical cognition research, however it is nevertheless likely that a non-negligible proportion of results will be difficult to replicate.

\section{Acknowledgements}

383 We would like to thank Edward Legg for carrying out a reproducibility check of our code.

\section{Author Contributions}

385 BGF and LO conceived of the study. All authors provided critical feedback on the methods 386 before data collection, BGF, DA, JF, JvdM and CT were primary coders and LO, SP and AV 387 were secondary coders. BGF wrote the manuscript and all authors were involved in interpreting 388 the data and editing. LO and NSC provided supervision.

\section{Conflict of Interest Statement}

390 The authors report no conflicts of interest in the publication of this manuscript. 
392

393

394

395

396

397

398

399

400

401

402

403

404

405

406

407

408

409

410

411

412

413

414

415

416

417

\section{References}

Baker, M. (2016). 1,500 scientists lift the lid on reproducibility. Nature News, 533, 452. https://doi.org/10.1038/533452a

Begley, C. G., \& Ellis, L. M. (2012). Drug development: Raise standards for preclinical cancer research. Nature, 483, 531-533. https://doi.org/10.1038/483531a

Benjamin, D. J., Berger, J. O., Johannesson, M., Nosek, B. A., Wagenmakers, E.-J., Berk, R., Bollen, K. A., Brembs, B., Brown, L., Camerer, C., Cesarini, D., Chambers, C. D., Clyde, M., Cook, T. D., De Boeck, P., Dienes, Z., Dreber, A., Easwaran, K., Efferson, C., ... Johnson, V. E. (2018). Redefine statistical significance. Nature Human Behaviour, 2, 6-10. https://doi.org/10.1038/s41562-017-0189-z

Button, K. S., A Ioannidis, J. P., Mokrysz, C., Nosek, B. A., Flint, J., J Robinson, E. S., \& Munafò, M. R. (2013). Power failure: Why small sample size undermines the reliability of neuroscience. Nature Publishing Group, 14, 365-376. https://doi.org/10.1038/nrn3475

Camerer, C. F., Dreber, A., Holzmeister, F., Ho, T.-H., Huber, J., Johannesson, M., Kirchler, M., Nave, G., Nosek, B. A., Pfeiffer, T., Altmejd, A., Buttrick, N., Chan, T., Chen, Y., Forsell, E., Gampa, A., Heikensten, E., Hummer, L., Imai, T., ... Wu, H. (2018). Evaluating the replicability of social science experiments in Nature and Science between 2010 and 2015. Nature Human Behaviour, 2, 637. https://doi.org/10.1038/s41562-018-0399-z

Chamberlin, T. C. (1965). The Method of Multiple Working Hypotheses: With this method the dangers of parental affection for a favorite theory can be circumvented. Science, 148, 754759. https://doi.org/10.1126/science.148.3671.754

Eaton, T., Hutton, R., Leete, J., Lieb, J., Robeson, A., \& Vonk, J. (2018). Bottoms-up! Rejecting Topdown Human-centered Approaches in Comparative Psychology. International Journal of Comparative Psychology, 31. Retrieved from https://escholarship.org/uc/item/11t5q9wt

Fanelli, D. (2012). Negative results are disappearing from most disciplines and countries. Scientometrics, 90, 891-904. https://doi.org/10.1007/s11192-011-0494-7 
Farrar, B., Boeckle, M., \& Clayton, N. (2020). Replications in Comparative Cognition: What Should We Expect and How Can We Improve? Animal Behavior and Cognition, 7, 1-22. https://doi.org/10.26451/abc.07.01.02.2020

Flis, I. (2019). Psychologists psychologizing scientific psychology: An epistemological reading of the replication crisis. Theory \& Psychology, 29, 158-181. https://doi.org/10.1177/0959354319835322

Hedges, L. V. (1984). Estimation of Effect Size under Nonrandom Sampling: The Effects of Censoring Studies Yielding Statistically Insignificant Mean Differences. Journal of Educational Statistics, 9, 61. https://doi.org/10.2307/1164832

Ioannidis, J. P. A. (2018). Meta-research: Why research on research matters. PLOS Biology, 16, e2005468. https://doi.org/10.1371/journal.pbio.2005468

Lakens, Daniel. (2017). Professors Are Not Elderly: Evaluating the Evidential Value of Two Social Priming Effects through P-Curve Analyses [Preprint]. PsyArXiv. https://doi.org/10.31234/osf.io/3m5y9

Lakens, Daniël. (2017). Equivalence Tests: A Practical Primer for t Tests, Correlations, and MetaAnalyses. Social Psychological and Personality Science, 8, 355-362. https://doi.org/10.1177/1948550617697177

LeBel, E. P., Berger, D., Campbell, L., \& Loving, T. J. (2017). Falsifiability is not optional. Journal of Personality and Social Psychology, 113, 254-261. https://doi.org/10.1037/pspi0000106

Maes, E., Boddez, Y., Alfei, J. M., Krypotos, A.-M., D’Hooge, R., De Houwer, J., \& Beckers, T. (2016). The elusive nature of the blocking effect: 15 failures to replicate. Journal of Experimental Psychology. General, 145, e49-71. https://doi.org/10.1037/xge0000200

Makel, M. C., Plucker, J. A., \& Hegarty, B. (2012). Replications in Psychology Research: How Often Do They Really Occur? Perspectives on Psychological Science, 7, 537-542. https://doi.org/10.1177/1745691612460688

Nissen, S. B., Magidson, T., Gross, K., \& Bergstrom, C. T. (2016). Publication bias and the canonization of false facts. ELife, 5, e21451. https://doi.org/10.7554/eLife.21451 
Open Science Collaboration, O. S. (2015). PSYCHOLOGY. Estimating the reproducibility of psychological science. Science, 349, aac4716. https://doi.org/10.1126/science.aac4716

Platt, J. R. (1964). Strong Inference: Certain systematic methods of scientific thinking may produce much more rapid progress than others. Science, 146(3642), 347-353. https://doi.org/10.1126/science.146.3642.347

Poulin-Dubois, D., Rakoczy, H., Burnside, K., Crivello, C., Dörrenberg, S., Edwards, K., Krist, H., Kulke, L., Liszkowski, U., Low, J., Perner, J., Powell, L., Priewasser, B., Rafetseder, E., \& Ruffman, T. (2018). Do infants understand false beliefs? We don't know yet - A commentary on Baillargeon, Buttelmann and Southgate's commentary. Cognitive Development, 48, 302 315. https://doi.org/10.1016/j.cogdev.2018.09.005

Povinelli, D. J. (2000). Folk physics for apes: The chimpanzee's theory of how the world works. Oxford University Press.

Rosenthal, R. (1979). The file drawer problem and tolerance for null results. Psychological Bulletin, 86, 638-641. https://doi.org/10.1037/0033-2909.86.3.638

Scheel, A. M., Schijen, M., \& Lakens, D. (2020). An excess of positive results: Comparing the standard Psychology literature with Registered Reports [Preprint]. PsyArXiv. https://doi.org/10.31234/osf.io/p6e9c

Sena, E. S., Worp, H. B. van der, Bath, P. M. W., Howells, D. W., \& Macleod, M. R. (2010). Publication Bias in Reports of Animal Stroke Studies Leads to Major Overstatement of Efficacy. PLOS Biology, 8, e1000344. https://doi.org/10.1371/journal.pbio.1000344

Simonsohn, U., Nelson, L. D., \& Simmons, J. P. (2014). P-curve: A key to the file-drawer. Journal of Experimental Psychology: General, 143, 534-547. https://doi.org/10.1037/a0033242

Smith, P. L., \& Little, D. R. (2018). Small is beautiful: In defense of the small-N design. Psychonomic Bulletin \& Review, 25, 2083-2101. https://doi.org/10.3758/s13423-018-1451-8

Stevens, J. R. (2017). Replicability and Reproducibility in Comparative Psychology. Frontiers in Psychology, 8, 862. https://doi.org/10.3389/fpsyg.2017.00862

Zwaan, R. A., Etz, A., Lucas, R. E., \& Donnellan, M. B. (2018). Making replication mainstream. Behavioral and Brain Sciences, 41, e120. https://doi.org/10.1017/S0140525X17001972 
Zwaan, R. A., Pecher, D., Paolacci, G., Bouwmeester, S., Verkoeijen, P., Dijkstra, K., \& Zeelenberg, R. (2018). Participant Nonnaiveté and the reproducibility of cognitive psychology. Psychonomic Bulletin \& Review, 25, 1968-1972. https://doi.org/10.3758/s13423-017-1348-y 


\section{Appendix} simulations:

480

$\begin{array}{lll}\text { Power } & \text { Population 1 } & \text { Population 2 } \\ 80 \% & X \sim N(50,5) & X \sim N(52.02,5) \\ 20 \% & X \sim N(50,5) & X \sim N(50.81,5) \\ 5 \% & X \sim N(50,5) & X \sim N(50,5)\end{array}$

The difference between Population 1 and Population 2 was calculated in order to give the desired power for a two-tailed two sample $t$-test with $\mathrm{n}=50$ per group.

100,000 samples were then taken from each Population and compared to each other, and the $p$ values under .05 were plotted in Figure 2 alongside the $p$-values we sampled from the physical cognition literature.

For Figure 3, the p-values we sampled from the physical cognition literature were plotted 487 alongside a mixture model of research at $80 \%$ power (1/12 of all research), $20 \%$ power (2/3 of all research) and $5 \%$ power (1/4 of all research) from the same populations as Figure 2. 